\title{
Design and Development of High Efficiency 150 kW Very Compact PLA Core Electromagnetic Coupler for Highly Resonant Power Transfer Technology
}

\section{Fatima Zahra Boudara1, Marc Rivaletto' ${ }^{1}$, Laurent Pecastaing ${ }^{*}$, Antoine Silvestre de Ferron ${ }^{1}$, Sylvain Paquet ${ }^{2}$, Jean-Pierre Brasile ${ }^{2}$}

${ }^{1}$ Univ Pau \& Pays Adour/E2s Uppa, Laboratoire Des Sciences De L’ingénieur Appliquées A La Mécanique Et Au Génie Electrique, Federation Ipra, Ea4581, Pau, France

${ }^{2}$ Effitech, Pau, France

Email: *laurent.pecastaing@univ-pau.fr

How to cite this paper: Boudara, F.Z., Rivaletto, M., Pecastaing, L., de Ferron, A.S., Paquet, S. and Brasile, J.-P. (2020) Design and Development of High Efficiency 150 kW Very Compact PLA Core Electromagnetic Coupler for Highly Resonant Power Transfer Technology. Journal of Electromagnetic Analysis and Applications, 12, 131-144. https://doi.org/10.4236/jemaa.2020.129011

Received: September 8, 2020

Accepted: September 27, 2020

Published: September 30, 2020

Copyright $\odot 2020$ by author(s) and Scientific Research Publishing Inc. This work is licensed under the Creative Commons Attribution International License (CC BY 4.0).

http://creativecommons.org/licenses/by/4.0/

\begin{abstract}
Highly Resonant Power Transfer (HRPT) technology is currently receiving very significant attention from the industry and the smart power grid distribution community in particular. This technology ensures electrical power transmission between two points while controlling the level of transmitted power and ensures the immediate shutdown of the transmitted power in the event of a problem. This paper reviews the inductive power transfer method and describes the design of an ultra-compact PLA core electromagnetic coupler. The proposed architecture confines the magnetic field in a toroidal PLA core transformer, and by avoiding the use of heavy and bulky shielding plates, reduces magnetic losses and avoids the Curie point. As a result, the overall unit has a weight of $5 \mathrm{~kg}$ and a volume of only $0.013 \mathrm{~m}^{3}$. The electromagnetic coupler is capable of transferring a peak power of $150 \mathrm{~kW}$ with an operating frequency of $193 \mathrm{kHz}$, giving a satisfactory efficiency of 95\%. The proposed novel system was first investigated through CST 3D numerical modelling to determine the electrical parameters of the coupler's equivalent circuit and its efficiency, to verify its compatibility with the ICNIRP 2010 standard and to evaluate its temperature rise with an air-cooling system. Afterwards, the designed coupler was built with a 3D printing device and finally tested experimentally. Simulation and experimental results are compared and show a good agreement.
\end{abstract}

\section{Keywords}

PLA Core, Toroidal Coupler, Resonant System, Low Stray Emission, Smart Grid 


\section{Introduction}

In toroidal coupler, the winding occupies the full periphery of the core, resulting in reduced leakage inductance and stray electromagnetic field. Besides, it has advantages related to size and volume. Thanks to those merits, it is widely used in low power applications but is still not very available in high power ones and less studied compared to the conventional transformer. Technically, the aim of this paper is to demonstrate that toroidal electromagnetic coupler can be used in modern high power applications, such as smart grid technology.

One of the main components of this technology is the transformer, which must ensure efficient power transmission with high-performance control and communication systems. So, in this present, we will show the possibility of replacing a conventional transformer by a galvanically isolated PLA core toroidal electromagnetic coupler that will address the main challenges of smart grid technology using the inductive transmission method.

Figure 1 shows a diagram of the power grid distribution. The power plant produces electricity. Through the step-up voltage transformer, the voltage is increased and power is directed to transmission systems that supply this power to customers from the distribution substation. Smart grid technology enables better coordination of local needs and capabilities while maximizing system reliability, resilience and stability [1].

In this context, the goal of this paper is to study the development of a very compact PLA core electromagnetic coupler that can be integrated in a voltage converter ensuring a galvanic isolation between the input and the output of the system. The design of a lightweight structure and a high-efficiency system are the key elements taken into account.

First the power transfer system is presented. Secondly, the electrical characteristics of the designed coupler are discussed in detail through the simulation results. Next, the prototype of the toroidal electromagnetic coupler and the experimental results are presented in the fourth section. Finally, in order to comply with international guidelines, electromagnetic leakage fields (EMLF) around the coupler are simulated, measured and discussed in the last section.

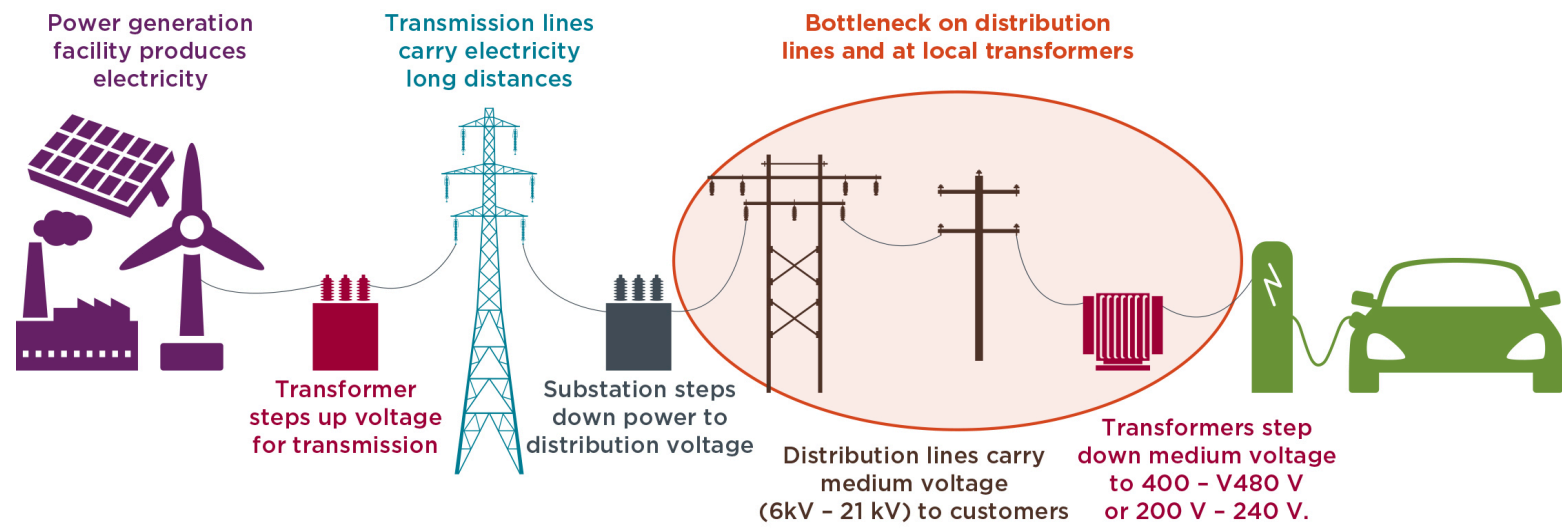

Figure 1. Schematic of the power grid distribution [1]. 


\section{Power Transfer System Overview}

A power transmission system block diagram is shown in Figure 2. It consists of a DC/AC power converter, two resonant compensation networks for the primary and secondary coils and a rectifier converter on the receiver side.

In a conventional transformer, the primary and secondary coils share the same magnetic core. Thus, the magnetic flux generated by the primary moves forward to the secondary, allowing a high coupling factor and good efficiency. On the other hand, in an electromagnetic PLA core coupler, the primary and secondary coils are not physically connected. Therefore, a small amount of magnetic flux generated by the primary flows through the secondary. This results in a poor coupling factor, in the range of 0.1 to 0.3 . This coefficient decreases as the air gap between the primary and secondary coils increases.

The differential Equation (1) and Equation (2) for the coupler are presented by the main components of the equivalent circuit in Figure 3.

$$
\begin{aligned}
& U_{1}=L_{1} \cdot \frac{\mathrm{d} i_{1}}{\mathrm{~d} t}+M \cdot \frac{\mathrm{d} i_{2}}{\mathrm{~d} t}+R_{1} \cdot i_{1} \\
& U_{2}=L_{2} \cdot \frac{\mathrm{d} i_{2}}{\mathrm{~d} t}+M \cdot \frac{\mathrm{d} i_{1}}{\mathrm{~d} t}+R_{2} \cdot i_{2}
\end{aligned}
$$

The inductances $L_{1}$ and $L_{2}$ are the primary and secondary self-inductances and $M$ is the mutual inductance between these two coils. $R_{1}$ and $R_{2}$ are the primary and secondary resistances of the wires that characterize the Joule losses. The coupler voltages on the primary and secondary sides are denoted respectively $U_{1}$ and $U_{2}$, while $i_{1}$ and $i_{2}$ are the coupler currents.

For the ideal transformer, the two windings are crossed by the same magnetic flux, therefore $M=\sqrt{L_{1} \cdot L_{2}}$. However, for the PLA core electromagnetic coupler, the magnetic leakage inductance $L_{\sigma}$ leads to $M<\sqrt{L_{1} \cdot L_{2}}$. That is why the coupling factor $k$ is defined by:

$$
k=\frac{M}{\sqrt{L_{1} \cdot L_{2}}}
$$



Figure 2. Block diagram of the power transmission system.

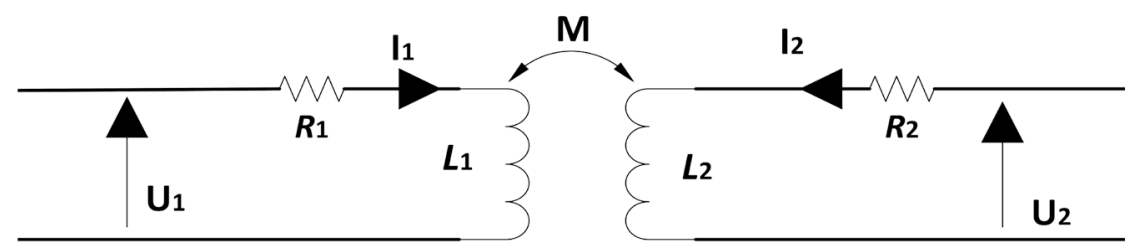

Figure 3. The coupler equivalent circuit. 
The weak value of the coupling factor in a PLA core electromagnetic coupler results in an increase in the leakage inductance value, so the voltage drop is increased and the magnetizing inductance is decreased. In this way, the magnetizing current is higher. Thus, additional reactive power must be supplied from the voltage source to the load for the same amount of active power, resulting in a significant reduction in efficiency. To compensate for the inductive effect and to ensure efficient power transfer, resonance capacitors are connected in series or parallel with the primary and secondary coils giving rise to four possible configurations (Series-Series, Series-Parallel, Parallel-Series, Parallel-Parallel). This double compensation is essential because the compensation on one side does not take into account the reactive energy linked to the other side. Besides, the selection of the compensation topology should mainly depend on the proposed application and the topology of the power converter chosen [2] [3]. To ensure an efficient energy transfer from the transmitter to the receiver, the capacitances of the primary $\left(C_{1}\right)$ and secondary $\left(C_{2}\right)$ compensations must be chosen such that the resonance frequency indicated by Equation (4) is equal to the switching frequency of the electronic power converter.

$$
f_{0}=\frac{1}{2 \pi} \cdot \frac{1}{\sqrt{L_{1} \cdot C_{1}}}=\frac{1}{2 \pi} \cdot \frac{1}{\sqrt{L_{2} \cdot C_{2}}}
$$

So, as a first step in the design of the Power Transmission System, a coil design as well as a suitable compensation topology should be selected. This will be discussed in the next section.

\section{3D Numerical Simulation Results}

The values of the primary and secondary currents provide the initial information for the selection of the characteristics of the wires of the coils. The cross-section of the conductor is chosen as a function of the current density. In our case, a density of $10 \mathrm{~A} / \mathrm{mm}^{2}$ is considered an acceptable benchmark. The conductor cross-section also influences the coil resistances and the self-inductances. In addition, the selection of the section must deal with aspects of cost and mechanical realization.

The maximum RMS current supplied by the Mosfets of the power converter is $400 \mathrm{~A}$, so four Litz wires in parallel with a cross-section of $10 \mathrm{~mm}^{2}$ are mandatory in order to be able to withstand this value of current without destroying the Litz wire. Therefore, a Litz wire consisting of $2500 \times 71 \mu \mathrm{m}$ strands and an outer diameter of $5 \mathrm{~mm}$ with a cross-section of $9.98 \mathrm{~mm}^{2}$ was selected for the primary and secondary windings. The diameter of the strands was chosen based on the skin depth at the operating frequency. The maximum current value also allowed us to estimate the minimum allowed value of the self-inductances of the electromagnetic coupler and therefore the length of the Litz wire.

The compactness of the system is the key element considered when sizing the toroidal coupler; for this reason, a spacing of $1 \mathrm{~cm}$ between the loops of the toroidal coupler was chosen. This distance corresponds to the minimum possible distance between loops without mechanical contacts and allows us to obtain a maximum coupling coefficient. 
Taking all this into account, a toroidal electromagnetic coupler was designed after a long optimization process carried out by our industrial partner. The outer diameter of the electromagnetic coupler is $248 \mathrm{~mm}$. The primary and secondary windings have the same loop diameter of $110 \mathrm{~mm}$. They consist of nine turns. This project does not only target the civilian market, but also the military one. To meet its needs in terms of system compactness, a resonant frequency of around $200 \mathrm{kHz}$ was selected. This frequency differs from the usual working frequency found in the literature, which generally does not exceed $85 \mathrm{kHz}$. The parameters of the selected design are listed in Table 1.

Using CST 3D numerical software, the toroidal coupler was simulated to determine the electrical parameters of the coupler's equivalent circuit. The diameter of a single strand of the Litz wire in high frequency applications is very small (a few tens of micrometers). Taking into account the depth of the skin effect requires substantial meshing and is time-consuming. Therefore, we used the method proposed by [4] to simulate the Litz wire.

The modeled coupler with EMLF distribution is presented in Figure 4. The primary and secondary windings wound over the entire surface of the toroid confine the magnetic field lines, offering the toroidal coupler very reduced electromagnetic radiation. The circuit parameters obtained from the CST software are presented in Table 2 in the next section.

As mentioned earlier, to overcome the issue of large leakage inductance, resonant capacitors are used. Numerous studies have been conducted to compare the four basic topologies [5] [6] [7]. It has been found that the Series-Series (SS) topology induces the lowest current in power electronics systems and prevents the destruction of components in the event of undesirable overvoltage. Besides, according to [8] [9] [10] the SS topology is the main compensation method often used in power transmission systems because it is the only configuration that does not vary the resonant frequency with coupling factor or load. Given these main advantages over other compensation methods, the SS topology was found to be the most suitable for our application and will therefore be used.

The overall system including the 3D model of the coupler was simulated using CST Design Studio with an equivalent load of $1.85 \Omega$, as shown in Figure 5.

The maximum input power obtained is $152 \mathrm{~kW}$ and the maximum output power is $147 \mathrm{~kW}$ with an approximate efficiency of $96.4 \%$ using the SS compensation topology.

For the thermal management of the power losses in the electromagnetic coupler, a forced-air cooling system is considered. The heat transfer coefficient needs to be determined for the Litz wire and for the PLA material. This coefficient empirically reflects the heat exchange from the inside (Litz wire) to the outside (forced air) and its value depends essentially on the type of material and the fluid flow. So, a theoretical thermal modelling of the hollow torus was carried out and the heat equation is solved in a torus coordinate system for the appropriate boundary conditions. The steady-state thermal problem is solved first and then the 
transient one. The calculated values for the surface heat transfer coefficients are $h_{\text {Litz_wire }}=245 \mathrm{~W} /\left(\mathrm{m}^{2} \cdot \mathrm{K}\right)$ for the Litz wire and $h_{\mathrm{PLA}}=150 \mathrm{~W} /\left(\mathrm{m}^{2} \cdot \mathrm{K}\right)$ for the PLA support with air flow of $25 \mathrm{~m} / \mathrm{s}$. Figure 6 shows the temperature distribution of the electromagnetic coupler in steady state at $150 \mathrm{~kW}$. The electromagnetic 3D simulation shows that the temperature is not too high for the Litz wire and for the PLA support. The maximum temperature reached respects the maximum thermal limits supported by the Litz wire, which is $120^{\circ} \mathrm{C}$, and the PLA, which is $60^{\circ} \mathrm{C}$.

The results predicted by the $3 \mathrm{D}$ modeling are very encouraging. A prototype of the electromagnetic coupler was then developed in order to experimentally verify the modeling results.

Table 1. Parameters of the selected design.

\begin{tabular}{ccc}
\hline Parameter & Variable & Value \\
Coupler diameter & $D_{\text {coil }}$ & $248 \mathrm{~mm}$ \\
Loop diameter & $D_{\text {loop }}$ & $110 \mathrm{~mm}$ \\
Number of turns & $N_{p} / N_{s}$ & $9 / 9$ \\
Litz wire diameter & $D_{\text {wire }}$ & $5 \mathrm{~mm}$ \\
Copper cross-section & $S_{\text {cu }}$ & $9.98 \mathrm{~mm}^{2}$ \\
Resonant frequency & $F_{\text {res }}$ & $193 \mathrm{kHz}^{2}$
\end{tabular}

Table 2. The measured and simulated electrical parameters.

\begin{tabular}{cccc}
\hline Parameter & Experimental value & Simulation value & Error \\
\hline$L_{1}, L_{2}$ & $1.50 \mu \mathrm{H}$ & $1.47 \mu \mathrm{H}$ & $2.0 \%$ \\
$M$ & $1.05 \mu \mathrm{H}$ & $1.1 \mu \mathrm{H}$ & $4.8 \%$ \\
$L_{\sigma}$ & $0.72 \mu \mathrm{H}$ & $0.66 \mu \mathrm{H}$ & $8.4 \%$ \\
$k$ & 0.72 & 0.74 & $2.8 \%$ \\
\hline
\end{tabular}



(a)

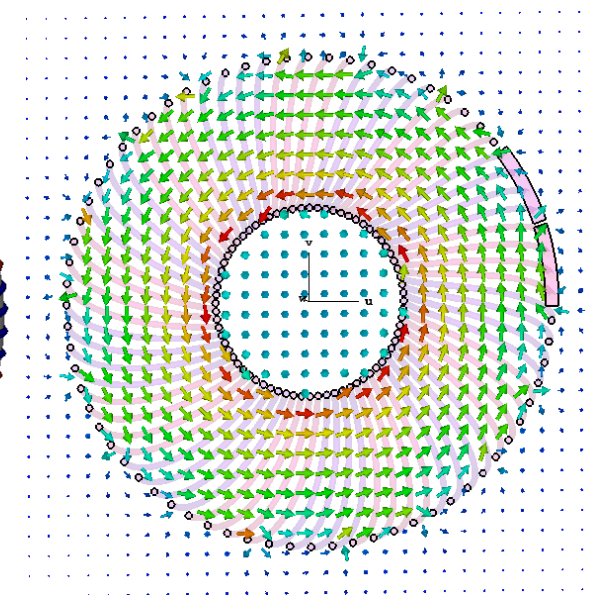

(b)

Figure 4. The toroidal coupler, the primary winding is represented by the red color and the secondary is represented by the blue (a) the coupler structure, (b) the EMLF distribution. 


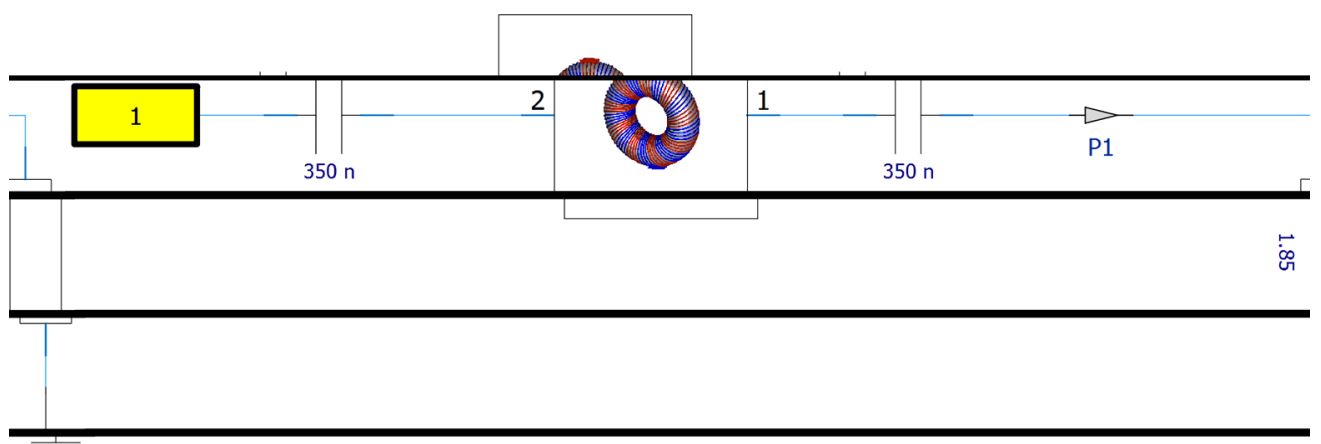

Figure 5. The simulation circuit combining the 3D model with circuit component.
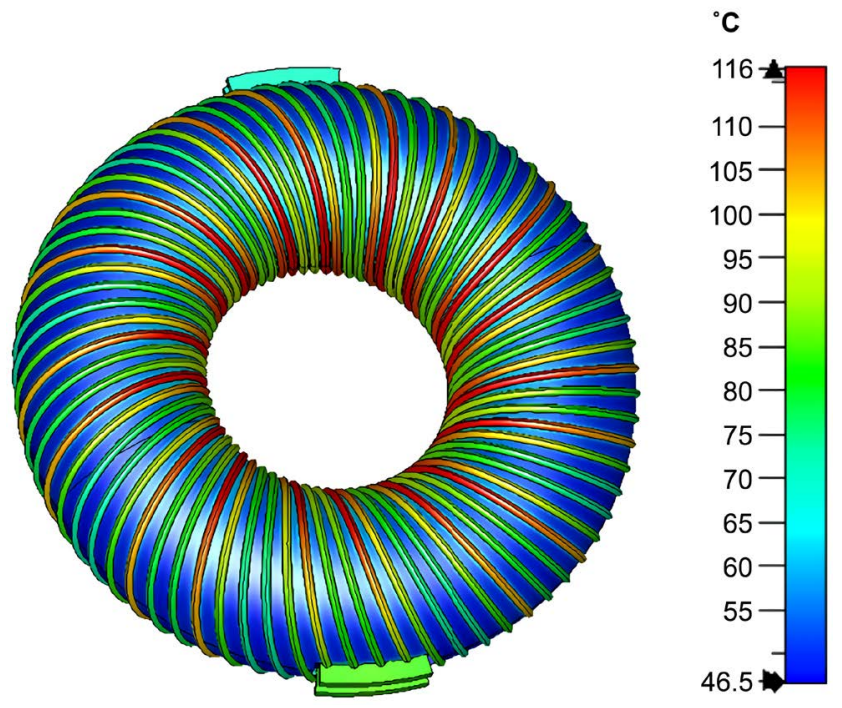

Figure 6. Temperature distribution of the electromagnetic coupler in steady state at $150 \mathrm{~kW}$.

\section{Experimental Tests and Validation}

Considering all the criteria discussed in the third section, a $150 \mathrm{~kW}$ HRPT system was designed and built. The operating frequency of $193 \mathrm{kHz}$ was determined and the SS topology was chosen as the compensation technique. To verify the efficiency of the proposed system, experimental tests were carried out. The experimental setup is shown in Figure 7.

\subsection{DC/AC Converter}

A 400 DC power supply is used for the measurements. Additionally, the energy storage is provided by (1ER300UAVT8) $300 \mu \mathrm{F} / 1.2 \mathrm{kV}$ type capacitors from SCR. Thanks to a capacitor bank composed of three of these capacitors, the energy delivered by the DC power supply is stored and then transmitted to the DC/AC converter. This system relieves the DC power supply during high power demands by preventing an excessive drop in the voltage it delivers. The DC/AC converter was built with $1.2 \mathrm{kV} / 300 \mathrm{~A} \mathrm{rms} \mathrm{SiC-MOSFETs}\left(R_{\mathrm{Ds}, \mathrm{on}}=4.2 \mathrm{~m} \Omega\right.$ at $25^{\circ} \mathrm{C}$ ) with a switching frequency of up to $200 \mathrm{kHz}$. The MOSFET high blocking voltage will allow future experiments with a higher DC voltage up to $1 \mathrm{kV}$. 


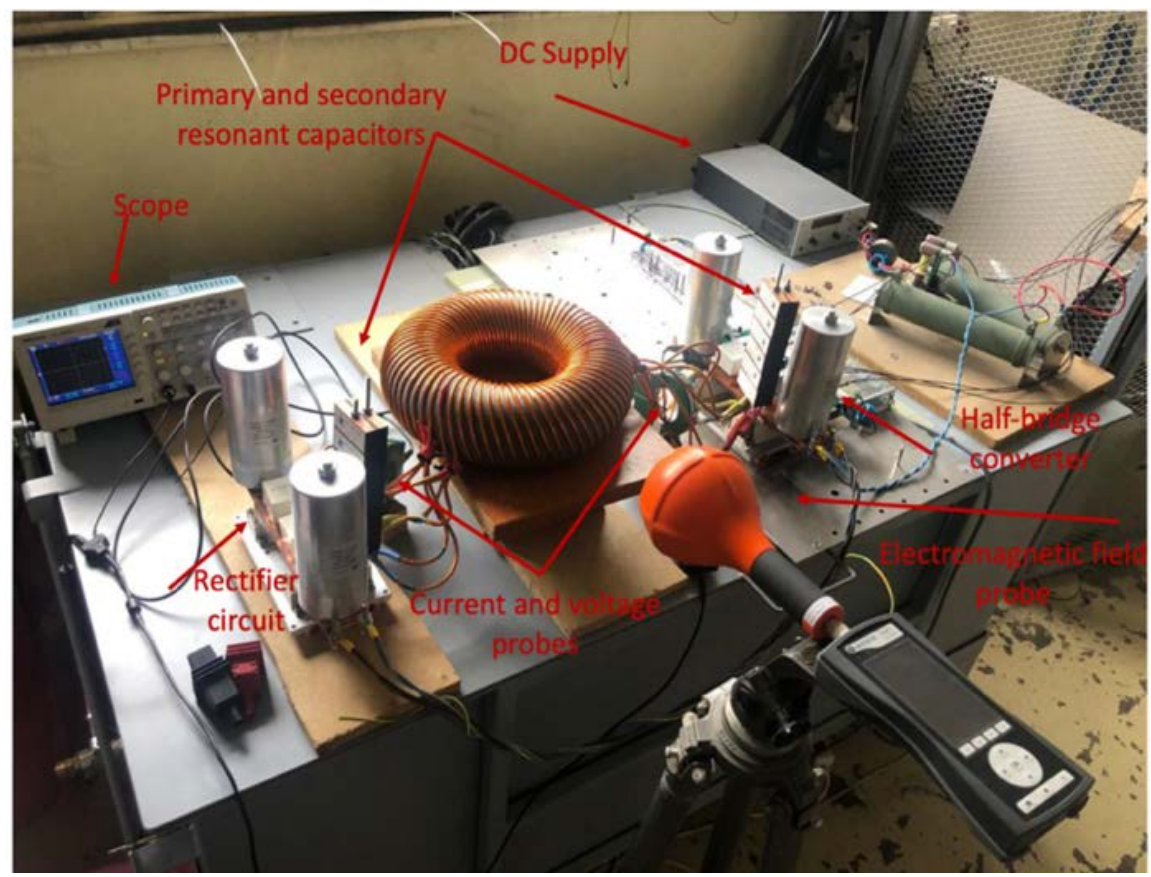

Figure 7. The experimental setup.

The converter includes an electronic board to control the H-bridge power converter and a measurement system is set up to adjust the real-time control of the system.

\subsection{Electromagnetic Coupler}

Based on the parameters of the coil design shown in Table 1, a prototype of the electromagnetic coupler was built. The cable holder was manufactured using a 3D printer.

As shown in Figure 8, the printable volume did not allow us to print the cable support in one piece, so we had to make the coupler in three sections. To prevent a high additional eddy current loss, the use of metals for the cable holder is not permitted. The PLA material was thus chosen to support the 3D printed cable holder because of its low-loss tangent and high-volume resistivity [11].

Table 2 shows a comparison between the measured and simulated equivalent circuit parameters with a calculation error with respect to the measured values. The measurements were performed using the RLC meter from HIOKI IM 3536 and demonstrate good agreement between the simulation and the practical implementation. The maximum difference between simulation and measurements for inductances is $4.8 \%$ and less than $2.8 \%$ for the coupling factor. The highest error appears for the leakage inductance because it is calculated according to $L_{\sigma}=\left(1-k^{2}\right) L_{1}$, so the error in the self-inductance and the coupling factor are added up. Besides, the high value of the coupling factor leads to a reduction in the leakage inductance. Overall, the results obtained are fully consistent with the intended performance of the designed coupler. 


\subsection{SS Compensation}

SS topology was chosen for the reasons discussed above. According to (4), capacitors of $350 \mathrm{nF}$ in the primary and secondary sides are required to ensure the highest efficiency of power transmission. As result, high-power resonant capacitors from Illinois Capacitor (104HC1102K2SM6) were used. According to the datasheet, the equivalent series resistance of the component at $200 \mathrm{kHz}$ is $1.5 \mathrm{~m} \Omega$.

\subsection{Rectifier Circuit}

A rectifier diode circuit is located at the output of the system. Fairchild RHRP30120 diodes $1.2 \mathrm{kV}, 300 \mathrm{~A}$ rms were chosen.

\subsection{Electromagnetic Coupler's Efficiency}

Figure 9 shows the waveforms of the DC/AC converter output voltage $\mathrm{U}_{1}$ and current $i_{1}$ (yellow and blue curves respectively) as well as the system output voltage $U_{2}$ and current $i_{2}$ (purple and green curves respectively). Currents were measured with Pearson 3972 current probes and voltages were measured with

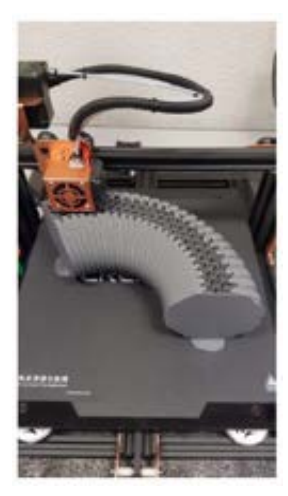

(a)



(b)

Figure 8. The 3D printed cable holder, (a) during the printing process, (b) the whole coupler.



Figure 9. Waveforms at the transmission at $150 \mathrm{~kW}$. 
TESTEC TT-SI 9010 A differential voltage probes. The measured input peak power is $155 \mathrm{~kW}(420 \mathrm{~V}, 368 \mathrm{~A})$ and the output peak power is measured to be $146 \mathrm{~kW}(424 \mathrm{~V}, 344 \mathrm{~A})$, thus demonstrating an efficiency of $94.4 \%$.

\section{Human Safety Concerns and International Regulations}

To enable the application of any electromagnetic coupler and to comply with international guidelines, the magnitude of EMLF must be checked. For this reason, several solutions have been proposed in the literature in order to limit the emission of the electromagnetic field [12] [13] [14]. Most of these studies use the active shielding method in order to better confine EMLF noise. In the OLEV system [15], a shielding method using a reactive resonant current loop in which the original magnetic field is cancelled by the magnetic field generated by the resonant loop current is adopted. These solutions have proven to be effective in ensuring low EMLF levels. But this makes the system more cumbersome and adds complications to the design of the coupler.

The unshielded toroidal structure of the PLA coupler has the merits of confining the EMLF inside the torus and keeping the system lightweight, as will be demonstrate in this paragraph.

Two international groups have set guidelines for human exposure to variations in flux density. The first is the International Committee on Electromagnetic Safety (ICES) under the leadership of the Institute of Electrical Electronic Engineers (IEEE) [16]. The second is the International Commission on Non-Ionizing Radiation Protection (ICNIRP) [17]. These regulations have been drawn up to limit human exposure to variations in flux density in order to prevent adverse effects on human health.

In the IEEE C95.1-2005 standards, the maximum permissible exposure of the head and torso is $205 \mu \mathrm{T}$ for public exposure and $615 \mu \mathrm{T}$ for occupational exposure. The limit value is even higher for the limbs, with $1130 \mu \mathrm{T}$. The latest published version of the ICNIRP [17] states that for a frequency range from $3 \mathrm{kHz}$ to $10 \mathrm{MHz}$, people should not be exposed to a flux density greater than $27 \mu \mathrm{T}$ for public exposure and $100 \mu \mathrm{T}$ for occupational exposure.

Compared with IEEE C95.1-2005, the ICNIRP 2010 standards are more stringent. Moreover, as mentioned in [18], the standards do not explicitly describe the measurement techniques for determining whether systems comply with the guidelines, thus making the development of the coupler's prototype difficult.

In order to verify compliance with international standards, simulations and measurements were undertaken to analyze leakage EMLF around the system. Simulations were carried out with the CST software and measurements were performed with a SMP2 WAVE CONTROL three-axis probe.

The evolution of the stray field during transmission of $150 \mathrm{~kW}$ at $193 \mathrm{kHz}$, together with the $\mathrm{X}$ and $\mathrm{Z}$ axes used (Figure 10(a)) for the reference simulation and measurement, is shown in Figure 10. The probe is moved along the $\mathrm{X}$ horizontal axis in Figure 10(b) and the $\mathrm{Z}$ vertical axis in Figure 10(c). The origin of the measurement is located in the center of the electromagnetic coupler. 


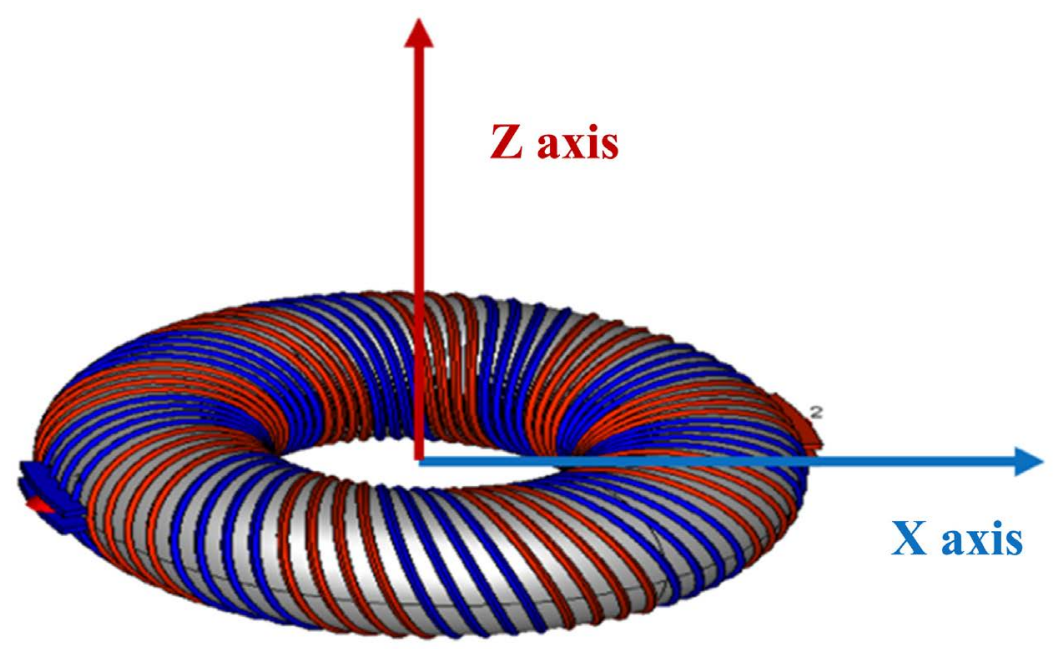

(a)

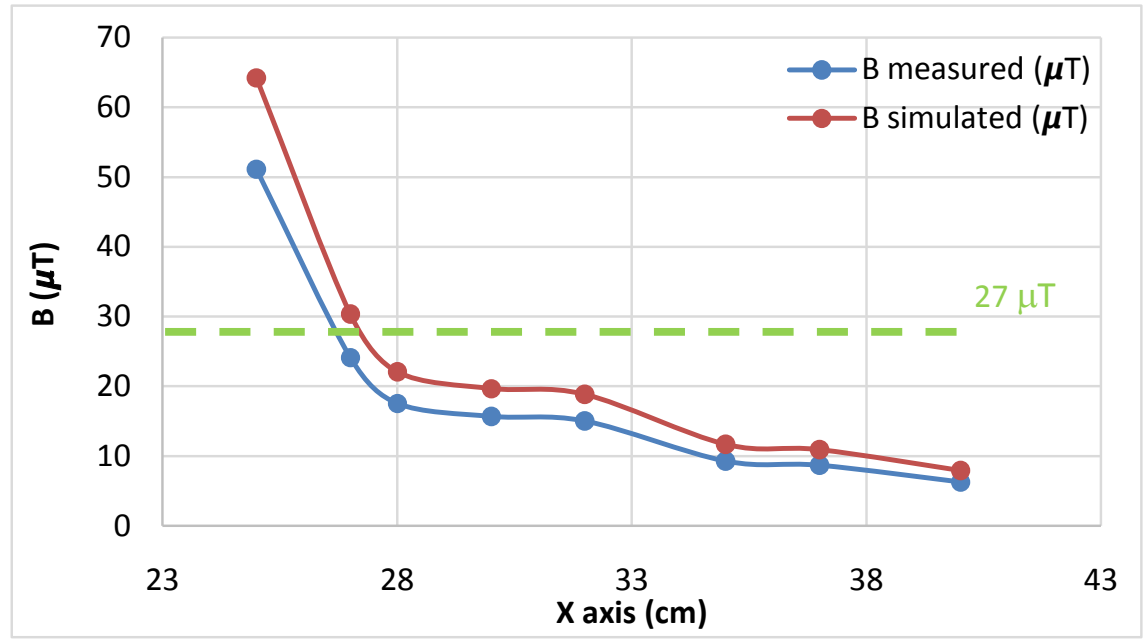

(b)

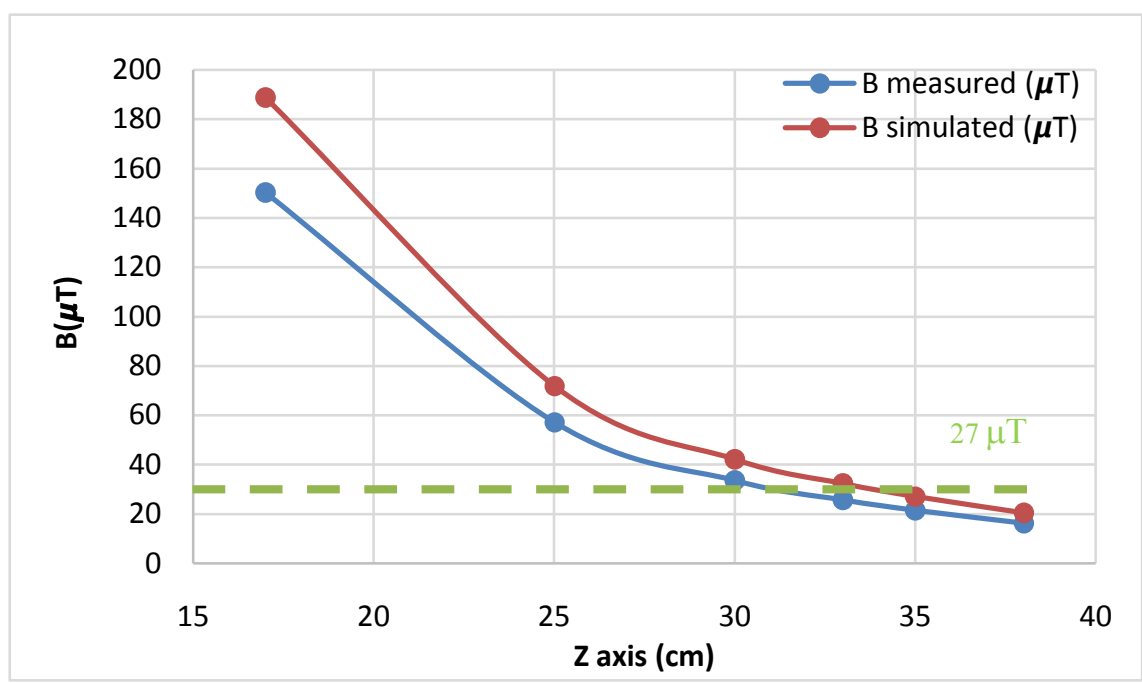

(c)

Figure 10. Evolution of unshielded induction B, (a): The axes used for the reference measurement (b): along $\mathrm{X}$ axis, (c): along $\mathrm{Z}$ axis. 
According to Figure 10(b) and Figure 10(c), for both axes, the simulation results predict the induction $B$ values slightly higher than those found by experimental measurement. This difference can be explained by the fact that the simulation is simplified and doesn't take into consideration all the parasitic elements found in the practical setup. In addition, the induction $B$ is measured to be $27 \mu \mathrm{T}$ at a distance of $26 \mathrm{~cm}$ for the $\mathrm{X}$ axis and $32 \mathrm{~cm}$ for the $\mathrm{Z}$ axis. By simulation, this value of EMLF is found at a distance of $28 \mathrm{~cm}$ for the $\mathrm{X}$ axis and 35 $\mathrm{cm}$ for the $\mathrm{Z}$ axis.

Despite this slight difference, in general, the results show a good match between simulation and measurement with a maximum related error of $25 \%$. This error is due both to the measurement uncertainty of the probe, which is $5 \%$, and to the accuracy of its position, which is estimated at $+/-1 \mathrm{~cm}$.

Those values are consistent with the safety guidelines recommended by the ICNIRP 2010 and IEEE C95.1-2005 for public exposure. Hence, the use of the representative electromagnetic coupler is safe.

\section{Conclusions}

In this paper, a very compact PLA core electromagnetic coupler which occupies an overall volume of $0.013 \mathrm{~m}^{3}$ was presented, designed, simulated, built and tested. In order to achieve a high magnetic coupling factor and low emission of stray radiation, a toroidal geometry was chosen. Furthermore, to enhance the power transmission capability, a SS compensation topology was considered. Based on the $3 \mathrm{D}$ simulation and the experimental implementation, the electromagnetic coupler designed demonstrates high efficiency of $94.4 \%$ for transmitted peak power of $150 \mathrm{~kW}$ at $193 \mathrm{kHz}$.

The work presented also shows that the electromagnetic coupler is in line with the ICNIRP 2010 recommendations for electromagnetic field exposure of the general public at a horizontal distance of $26 \mathrm{~cm}$ and a vertical distance of $32 \mathrm{~cm}$ from the center of the coupler.

Actually, the electromagnetic coupler designed could transfer up to $1 \mathrm{MW}$ peak power. However, the thermal limit of PLA, which is $60^{\circ} \mathrm{C}$ does not allow the conduction of high-power tests. Working at this level of power requires more efficient cooling systems for the toroidal electromagnetic coupler or replacing the PLA material with other more heat resistant material such as TC poly which has a better temperature limit of $175^{\circ} \mathrm{C}$. The project team is still working on this aspect.

\section{Acknowledgements}

The authors gratefully acknowledge the French Ministry for Defense (Direction Générale de l'Armement) for its financial support for this work (RAPID CEMPER $\mathrm{N}^{\circ} 172906093$ ).

The authors would also like to thank the support team of CST Dassault system for their useful help and discussions along the simulation process. 


\section{Conflicts of Interest}

The authors declare no conflicts of interest regarding the publication of this paper.

\section{References}

[1] Nicholas, M. and Hall, D. (2018) Lessons Learned on Early Electric Vehicle Fast-Charging Deployments. International Council on Clean Transportation, Washington, USA, 54.

[2] Steigerwals, R.L., Saj, C.F. and Croff, G.A. (2001) Analysis and Design of a Contactless Rotary Power Transfer System. 2001 IEEE $32^{\text {nd }}$ Annual Power Electronics Specialists Conference, Vancouver, Canada, 17-21 June 2001, 2125-2130.

[3] Sallan, J., Villa, J.L., Llombart, A. and Sanz, J.F. (2009) Optimal Design of ICPT Systems Applied to Electric Vehicle Battery Charge. IEEE Transactions on Industrial Electronics, 56, 2140-2149.

[4] Etemadrezaei, M. and Lukic, S.M. (2012) Equivalent Complex Permeability and Conductivity of Litz Wire in Wireless Power Transfer Systems. 2012 IEEE Energy Conversion Congress and Exposition (ECCE), Raleigh, NC, 15-20 September 2012, 3833-3840.

[5] Ryu, M., Cha, H., Park, Y. and Back, J. (2005) Analysis of the Contactless Power Transfer System Using Modelling and Analysis of the Contactless Transformer. $31^{\text {st }}$ Annual Conference of IEEE Industrial Electronics Society, Raleigh, USA, 6-10 November 2005, 7 .

[6] Abdelhamid, E., AbdelSalam, A.K., Massoud, A. and Ahmed, S. (2014) An Enhanced Performance IPT Based Battery Charger for Electric Vehicles Application. 2014 IEEE $23^{\text {rd }}$ International Symposium on Industrial Electronics, Turkey, Istanbul, 1-4 June 2014, 1610-1615.

[7] Bhuyan, S., Sivanand, K. and Panda, S.K. (2013) Effect of Design Parameters on Resonant Wireless Energy Transfer System. Journal of Electromagnetic Waves and Applications, 27, 288-298. https://doi.org/10.1080/09205071.2013.744125

[8] Lee, J.-W., Woo, D.-G., Ryu, S.-H., Lee, B.-K. and Kim, H.-J. (2015) Practical Bifurcation Criteria Considering Coil Losses and Compensation Topologies in Inductive Power Transfer System. 2015 IEEE International Telecommunications Energy Conference (INTELEC), Osaka, Japan, 18-22 October 2015, 1-6.

[9] Chopre, S. and Bauer, P. (2013) Analysis and Design Considerations for a Contactless Power Transfer System. 2011 IEEE $33^{\text {rd }}$ International Telecommunications Energy Conference (INTELEC), Amsterdam, Netherlands, 9-13 October 2011, 1-6.

[10] Kalwar, K.A., Aamir, M. and Mekhilef, S. (2015) Inductively Coupled Power Transfer (ICPT) for Electric Vehicle Charging-A Review. Renewable and Sustainable Energy Reviews, 47, 462-474. https://doi.org/10.1016/j.rser.2015.03.040

[11] Veselý, P., Horynová, E., Tichý, T. and Šefl, O. (2018) Study of Electrical Properties of 3D Printed Objects. 5.

[12] Knecht, O., Bosshard, R., Kolar, J.W. and Starck, C.T. (2014) Optimization of Transcutaneous Energy Transfer Coils for High Power Medical Applications. 2014 IEEE 15 th Workshop on Control and Modeling for Power Electronics (COMPEL), Santander, Spain, 22-25 June 2014, 1-10.

[13] Kar, D.P., Biswal, S.S., Sahoo, P.K., Nayak, P.P. and Bhuyan, S. (2018) Selection of Maximum Power Transfer Region for Resonant Inductively Coupled Wireless Charging System. AEU-International Journal of Electronics and Communications, 84, 84-92. https://doi.org/10.1016/j.aeue.2017.11.023 
[14] Bosshard, R. (2015) Multi-Objective Optimization of Inductive Power Transfer Systems for EV Charging. ETH Zurich.

[15] Kim, J., Kong, J., Kim, S. and Suh, H. (2013) Coil Design and Shielding Methods for a Magnetic Resonant Wireless Power Transfer System. Proceedings of the IEEE, 101, 1332-1342.

[16] Institute of Electrical and Electronics Engineers (2016) IEEE Standard for Safety Levels with Respect to Human Exposure to Radio Frequency Electromagnetic Fields, 3 kHz to $300 \mathrm{GHz}$. IEEE C95. 1-1991, New York.

[17] International Commission on Non-Ionizing Radiation Protection (2009) ICNIRP Guidelines for Limiting Exposure to Time-Varying Electric, Magnetic and Electromagnetic Fields up 300GHz. Healthy Physics, 97, 257-258.

[18] Budhia, M., Covic, G.A. and Boys, J.T. (2011) Design and Optimization of Circular Magnetic Structures for Lumped Inductive Power Transfer Systems. IEEE Transactions on Power Electronics, 26, 3096-3108. 